\title{
Polymyositis as a Rare Musculoskeletal Manifestation of Chronic Graft-Versus-Host Disease: A Case Report of a 33-Year- Old Patient
}

David Ben-Nun. ${ }^{\top}$

\begin{abstract}
Background: Musculoskeletal manifestations of chronic Graft-Versus-Host Disease (GVHD) are rare and unfamiliar to most clinicians. Here we discuss the pathophysiology of chronic GVHD, current treatments and direction of research for improved therapy and prophylaxis and focus on the common and less common musculoskeletal complications of this unfortunately prevalent and burdensome disease. The Case: This is a case report of a 33-year-old male with a past medical history of Acute Myeloid Leukemia (AML) who presented with a 1-month history of generalized, proximal weakness and was found to have polymyositis secondary to chronic GVHD. Elicitation of further history showed that the patient had had multiple manifestations of both acute and chronic graft-versus-host disease in the two years following hematopoietic stem cell transplantation (HSCT). Conclusion: It is important for clinicians to be familiar with polymyositis secondary to chronic GHVD, which may appear in patients more than 100 days following allogenic HSCT and typically presents as diffuse, generalized myopathy with preserved sensation and elevated CPK and aldolase. The current mainstay of treatment is glucocorticoids with or without a calcineurin inhibitor, however due to the side effects associated with long term treatment, more effective prophylactic and therapeutic treatments are needed to address this and other manifestations of chronic GVHD.
\end{abstract}

Key Words: Polymyositis; Graft-Versus-Host Disease; Muscular Diseases; Acute Myeloid Leukemia; Hematopoietic Stem Cell Transplantation (Source: MeSHNLM).

\section{Introduction}

Graft-Versus-Host Disease (GVHD) is a pathological process that occurs when immune cells from a donor graft recognize host antigens as foreign and trigger an immune response. GVHD is the most common life-threatening complication of allogenic hematopoietic stem cell transplantation (HSCT) and is classified into acute and chronic based on the length of time after which the immune response occurs following transplantation. ${ }^{1}$ Acute GVHD is considered any manifestation of graft T cell immune activation within the first 100 days following HSCT. ${ }^{2}$ Typical signs and symptoms of acute GVHD include a maculopapular rash, hyperbilirubinemia with jaundice, nausea and vomiting, anorexia, and watery or bloody diarrhea with crampy abdominal pain. 3 By some estimates, incidence of acute GVHD may be as low as $9 \%$ or up to $50 \%$ of patients who undergo HSCT from Human Leukocyte Antigen (HLA)matched sibling donors, which indicates that it is a significant medical problem especially given the fact that HSCT is a relatively common procedure..$^{4-6}$ Between the years 2013-2017, the Center for International Blood and Marrow Transplant Research reported that 106,112 HSCT procedures were carried out in the United States. Out of that total, approximately 43,962 were allogeneic transplants, which is an average of 8,792 allogeneic HSCTs annually.

Chronic GVHD was first described in 1978 and has been defined as any alloimmunity that results in clinical manifestations that occur more than 100 days post HSCT.(2) Chronic GVHD occurs in 30 to $70 \%$ of patients who have undergone allogeneic HSCT of nonmanipulated donor grafts and have received standard prophylaxis with a calcineurin inhibitor and an antimetabolite.(1) Chronic GVHD can have protean manifestations that range from fibrotic skin disease resembling systemic sclerosis, bronchiolitis, salivary and lacrimal gland disease, and eosinophilic fasciitis. ${ }^{8}$

The pathophysiology of chronic GVHD is believed to involve three phases: early inflammation and tissue injury, chronic inflammation and dysregulated immunity, and aberrant tissue repair often accompanied by fibrosis.( 9 ) In the first phase, there is translocation of bacteria and fungi across epithelial tight junctions, which are believed to be made more porous as a result of tissue damage that may occur following cytotoxic cell conditioning with chemotherapy or radiotherapy during the process of HSCT. This porosity leads to the release of immunogenic molecules that are not normally found in the extracellular space, which triggers clonal expansion of $B$ cells and differentiation of $T$ cells into type 1, type 2, and type 17 helper T-cells. This expansion of T cells causes a concomitant increase in the amount of auto-reactive $T$ cells that escape immune regulation both in the thymus and in the periphery. Autoreactive $T$ cells, along with toxic effects of the conditioning regimen, prophylaxis with calcineurin inhibitors, and immunoglobulin deposition lead to further thymic injury which further degrades the immune system's ability to filter out autoreactive immune cells in the thymus. In the final phase, activated macrophages secrete fibrotic growth factors such as Transforming Growth Factor Beta (TGF$\beta$ ) and Platelet-Derived Growth Factor (PDGF), which stimulate fibroblasts to lay down extracellular collagen and causes widespread fibrosis and sclerosis in various organs.

In 2005, the National Institutes of Health (NIH) compiled a formal set of guidelines regarding diagnosis of chronic GVHD, which were updated in 2014, that stipulate that the diagnosis of chronic GVHD requires "at least 1 diagnostic manifestation of chronic GVHD or at least 1 distinctive

\footnotetext{
Medical Student, Sackler School of Medicine, Tel Aviv University, Tel Aviv, Israe
}

About the Author: David Ben-Nun is a fourth-year medical student at Sackler School of Medicine at Tel Aviv University in Israel. He recently matched to the University of Texas at Austin Dell Medical Center for a residency in internal medicine, which he will begin later this vear. He is currently involved in research on heart failure with preserved ejection fraction, among other topics, and hopes to pursue a fellowship in cardiology following residency.

Correspondence:

David Ben-Nun

Address: 35, Tel Aviv-Yafo, 6997801, Israel

Email: bennun@mail.tau.ac.il
Editor: Mihnea-Alexandru Căman Student Editors: Madeleine Jemima Cox Submission: Mar 28, 2020 Acceptance: Apr 10, 2020 Publication: Apr 25, 2020 Process: Peer-reviewed 
manifestation, with the diagnosis confirmed by pertinent biopsy, laboratory tests, or radiology in the same or another organ" along with distinction from acute GVHD and exclusion of other possible causes. ${ }^{4}$ The mainstay of treatment of chronic GVHD requiring systemic treatment is and has been glucocorticoids, along with a calcineurin inhibitor. If patients require additional therapy following the prolonged use of glucocorticoids, secondary agents including rituximab, cyclophosphamide, imatinib, mycophenolate mofetil, IL-2, extracorporeal phonophoresis, methotrexate, bortezomib and as well as other novel immunosuppressive treatments are used. ${ }^{1,10}$

Musculoskeletal manifestations that result from chronic GVHD are uncommon and likely to be missed by many clinicians who are unaware of this entity. The goal of reporting on the case below is to emphasize the importance of being aware of the presentation, diagnostic guidelines and recommended treatment of polymyositis secondary to chronic GVHD in patients who have undergone HSCT.

\section{The Case}

Here we report on the case of a 33-year-old male living in central Israel with a past medical history of Acute Myeloid Leukemia (AML) diagnosed two years prior who presented to his local hospital with a 1-month history of generalized weakness that was more pronounced in the lower body. The patient's chief complaint was that he was having difficulty accomplishing tasks of daily living such as lifting items around the house, getting out of bed, preparing food and driving. He had initially gone to see a neurologist in his community, who noted that he had profound upper and lower body weakness and referred him to the local hospital. The patient stated that the weakness had begun to progress more rapidly in the 10 days leading up to his presentation and that he now needed to use crutches in order to walk. He reported no sensory deficits or urinary incontinence. He also denied a family history of neurological or musculoskeletal disease, significant history of substance abuse, recent notable travel or sick contacts or occupational exposures to toxic chemicals.

The patient had been generally healthy prior to his diagnosis of AML two years prior. At that time, he had presented with complaints of fever, weakness, vertigo and headache for one week. Following an extensive work up, the patient was diagnosed with FMS-like tyrosine kinase 3 -internal tandem duplication (FLT3-ITD) positive AML, which is named for its similarity to a tyrosine kinase that binds macrophage or monocyte colony-stimulating factor and is encoded by the Feline McDonough Sarcoma (FMS) oncogene. "1 He was treated with a " $7+3$ " regimen of daunorubicin and cytarabine plus midostaurine as well as prophylactic antibiotics and steroids. The patient ultimately underwent allogenic HSCT two months after his initial presentation with a donor who was a 10/10 match (both alleles matched at HLA loci A, B, C, DRB1, and $\mathrm{DQB} 1$ ). One month following his HSCT, the patient was hospitalized with a febrile illness and found to have elevated CMV titers as well as elevated AST and ALT enzymes. He was treated at the time for a suspected viral infection with valganciclovir, piperacillin/tazobactam and prednisone. The patient later developed facial skin rashes and oral ulcers and his prednisone dose was increased. Soon afterward, he also suffered from a febrile illness with respiratory symptoms including cough and slight oxygen desaturation. He underwent bronchoscopy and a biopsy was taken from his respiratory tract, which was later found to be positive for Corynebacterium and he was treated with antibiotics. Later that same year, the patient presented with a complaint of gross hematuria and he was again prescribed prednisone and the hematuria eventually resolved.

At the patient's presentation for a chief complaint of generalized weakness, he was taking voriconazole, acyclovir, and trimethoprimsulfamethoxazole for antibacterial and antiviral prophylaxis as well as sorafenib for maintenance AML treatment. He was tapering his dose of prednisone and currently taking $5 \mathrm{mg}$ daily, which was a decrease from 6omg daily a few months prior.
At the hospital, the patient's vital signs showed that he was afebrile with a regular pulse of 85 , a blood pressure of $107 / 55$ and a respiratory rate within normal limits. The patient's extraocular muscle movements and visual fields were intact. Both arms and legs were hypotonic and patient could not hold them up against gravity, however no pronation was noticed. Arm muscle strength was rated as $4+/ 5$ bilaterally both proximal and distal. Wrist flexion was rated as $5 / 5$. In the lower body, hip flexion was rated as $3 / 5$ bilaterally, knee flexion was $4+/ 5$ bilaterally, knee extension was $4+/ 5$ bilaterally and foot plantar flexion and dorsiflexion was rated as $5 / 5$ bilaterally. The patient's reflexes were $2+$ throughout without any pathological reflexes. Superficial touch was intact in upper and lower body. The patient's gait was narrow-based with left foot dragging and he required a crutch in order to move around in his hospital room.

Laboratory results showed that the patient had a macrocytic anemia of $12.3 \mathrm{~g} / \mathrm{dL}$ with a MCV of 99.4 (normal 80-94), thrombocytopenia of 90 $\mathrm{K} / \mathrm{micl}$ (normal 130-400), leukocytosis of $13.32 \mathrm{~K} / \mathrm{micl}$ (normal 4.8-10.8) with eosinophilia of $4.3 \mathrm{~K} / \mathrm{micl}$ (normal $0.0-0.8$ ). All of these abnormal blood test findings were confirmed to have been noted on prior labs. The patient had CRP a $1.86 \mathrm{mg} / \mathrm{L}$ (normal 0.0 - 5.0) and ESR of 29 (normal $0.0-8.0$ ). Basic metabolic and coagulation panels were within normal limits. Patient's CPK and TSH were within normal limits. A lumbar puncture was performed which revealed an opening pressure of $12 \mathrm{~cm}$ of $\mathrm{H}_{2} \mathrm{O}, 1 \mathrm{WBC} / \mathrm{uL}$, no erythrocytes, glucose of $47 \mathrm{mg} / \mathrm{dL}$ and no xanthochromia. A flow cytometry test was negative for any abnormal cell populations in CSF and CSF culture and a Biofire panel for various microbiological pathogens was also negative. Patient serum and CSF was checked for antibodies including ANA, anti-dsDNA, anti-J01, antiScl-70, anti-voltage-gated calcium channel, anti-acetylcholine receptor, anti-GM-1 among others and were all negative. Hepatitis and HIV tests were also negative.

The patient was sent for a non-contrast head CT which showed a minor lesion in the right pons that was interpreted as an old finding that was likely an artifact.

The patient was also sent for electromyography (EMG), which revealed no spontaneous activity upon needle insertion and low amplitude, polyphasic motor unit action potentials with decreased duration along with increased recruitment in all nerves including the median, ulnar, peroneal, tibial and axillary nerves. Nerve conduction studies showed distal motor latency and conduction velocity were preserved for both sensory and motor components.

The patient was treated with 6omg of methylprednisolone IV and was referred to hematology for treatment of presumed polymyositis secondary to chronic GVHD. Muscle biopsy was also recommended in order to confirm the working diagnosis but deferred since it seemed reasonable to assume this was a manifestation of chronic GVHD given the patient's history and findings. Following a stay in the hematooncology department, the patient's dose of steroids was changed to 5omg daily of prednisone. The patient was instructed to continue taking prophylactic antibiotics and schedule a follow up appointment with his hematologist for continued treatment of chronic GVHD manifestations.

\section{Discussion}

This report details the case of a patient with a history of FLT3-ITD positive AML and acute and chronic GVHD who presented with progressive weakness of one month's duration and was diagnosed with presumed polymyositis secondary to chronic GVHD. According to the NIH Consensus Criteria on Chronic GVHD, polymyositis is a recognized diagnostic finding in chronic GVHD (Figure 1) and, in this case, it was confirmed via EMG, which clearly demonstrated a myopathic pattern in multiple nerves in the upper and lower body. Other causes of myopathy including prolonged steroid use, auto-immune disease, hypokalemia, hypothyroidism, infection and otherwise were ruled out and the patient's presentation timeframe of more than 100 days post HSCT excluded acute GVHD as a potential cause. 
Musculoskeletal manifestations of chronic GVHD are very rare and when present do not usually manifest as myositis. In a retrospective chart review of all cases of myositis that developed in 7161 patients who underwent HSCT at the Fred Hutchinson Cancer Research Center in Seattle, Washington between 1969 and 1999, only 12 patients out of $1859(0.6 \%)$ who developed chronic GVHD also developed myositis between 7 and 55 months after HSCT. ${ }^{12}$ In another case series of 381 patients with chronic GVHD, Parker at el. reported that myositis occurred in 11 patients out of $318(3.5 \%) .13$ The incidence of myositis in the first study at the Hutchinson Cancer Center was calculated to be 23 per 100,000 person-years for all HSCT patients and 49 per 100,000 person-years for HSCT patients who had previously reported chronic GVHD manifestations. ${ }^{22}$ For a comparison of prevalence in the general population, in a large study that looked at 35 million commercially insured individuals in the US, the incidence of idiopathic inflammatory myositis (including dermatomyositis, polymyositis and sporadic inclusion body myositis), was found to be between 5.8 to 7.9 per 100,000 person-years from 2003-2008. ${ }^{14}$

Figure 1. Section of Scoring Card from NIH Chronic Graft Versus Host Disease Criteria Relevant to Musculoskeletal Manifestations.

\begin{tabular}{|c|c|c|c|c|}
\hline & SCORE 0 & SCORE 1 & SCORE 2 & SCORE 3 \\
\hline $\begin{array}{l}\text { P-ROM score } \\
\text { (see below) } \\
\text { Shoulder }(1-7): \\
\text { Elbow }(1-7): \\
\text { Wrist/finger }(1-7): \\
\text { Ankle }(1-4):\end{array}$ & $\begin{array}{l}\square \text { No } \\
\text { symptoms }\end{array}$ & $\begin{array}{l}\text { Mild tightness of } \\
\text { arms or legs, } \\
\text { normal or mild } \\
\text { decreased range of } \\
\text { motion (ROM) } \\
\text { AND not affecting } \\
\text { ADL }\end{array}$ & $\begin{array}{l}\text { Tightness of arms or } \\
\text { legs OR joint } \\
\text { contractures, } \\
\text { erythema thought } \\
\text { due to fasciitis, } \\
\text { moderate decrease } \\
\text { ROM AND mild to } \\
\text { moderate limitation } \\
\text { of ADL }\end{array}$ & $\begin{array}{l}\text { Contractures WITH } \\
\text { significant decrease of } \\
\text { ROM } A N D \text { significant } \\
\text { limitation of ADL } \\
\text { (unable to tie shoes, } \\
\text { button shirts, dress self } \\
\text { etc.) }\end{array}$ \\
\hline $\begin{array}{l}\text { GENITAL TRACT } \\
\left.\text { (See Supplemental figure }^{\ddagger}\right) \\
\square \text { Not examined } \\
\text { Currently sexually active } \\
\square \text { Yes } \\
\square \text { No }\end{array}$ & $\square$ No signs & $\begin{array}{l}\text { Mild signs }{ }^{\ddagger} \text { and } \\
\text { females with or } \\
\text { without discomfort } \\
\text { on exam }\end{array}$ & $\begin{array}{l}\text { Moderate signs }{ }^{\ddagger} \text { and } \\
\text { may have } \\
\text { symptoms with } \\
\text { discomfort on exam }\end{array}$ & $\begin{array}{l}\square \text { Severe signs }{ }^{\ddagger} \text { with } \\
\text { or without } \\
\text { symptoms }\end{array}$ \\
\hline \multicolumn{5}{|c|}{$\square$ Abnormality present but explained entirely by non-GVHD documented cause (specify): } \\
\hline \multicolumn{5}{|c|}{$\begin{array}{l}\text { Other indicators, clinical features or complications related to chronic GVHD (check all that apply and assign a } \\
\text { score to severity (0-3) based on functional impact where applicable none }-0 \text {, mild }-1 \text { moderate }-2, \text { severe }-3 \text { ) }\end{array}$} \\
\hline$\square$ Ascites (serositis) & \multicolumn{2}{|c|}{$\square$ Myasthenia Gravis } & \\
\hline$\square$ Pericardial Effusion_ & \multicolumn{2}{|c|}{$\square$ Peripheral Neuropathy_ } & \multicolumn{2}{|c|}{$\square$ Eosinophilia $>500 / \mu \mathrm{l}$} \\
\hline$\square$ Pleural Effusion(s) & \multicolumn{2}{|c|}{$\square$ Polymyositis } & & ts $<100,000 / \mu 1$ \\
\hline$\square$ Nephrotic syndrome & $\square$ Weis & ht loss $>5 \% *$ without $\mathrm{G}$ & Others & (specify): \\
\hline $\begin{array}{l}\text { Overall GVHD Severity } \\
\text { (Opinion of the evaluator) }\end{array}$ & № G & $\square$ Mild & $\square$ Moderate & 口 Severe \\
\hline
\end{tabular}

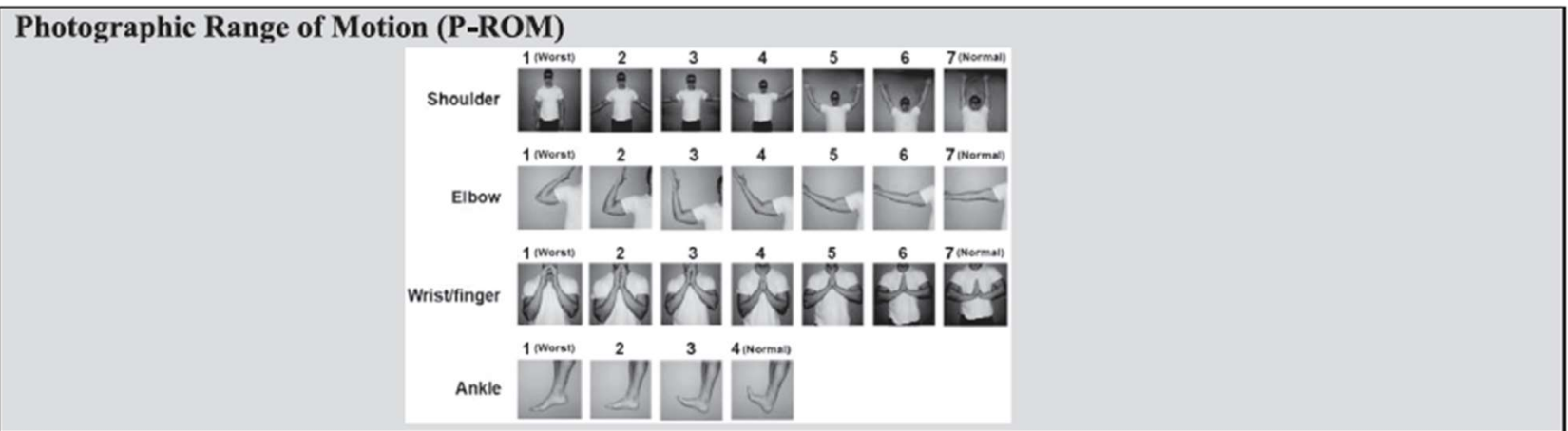

Legend. Section of organ system scoring card relevant to musculoskeletal complications of chronic GVHD as drafted by The National Institutes of Health Consensus Development Project on Criteria for Clinical Trials in Chronic Graft-versus-Host Disease in 2014. The scoring card focuses mostly on musculoskeletal changes related to joint contractures, fasciitis and their related manifestations with a photographic range of motion scale provided for ranking perceived joint range of motion. There is also a box that can be ticked with a related severity scale of $1-3$ in case of noted polymyositis. ${ }^{15}$ 
In patients who do develop musculoskeletal complications of chronic GVHD, the most common manifestation is fasciitis that is characterized by sclerosis of the overlying skin and subcutaneous tissues causing joint contractures, edema and restricted range of motion. ${ }^{15}$ Figure 1 shows the section of the scoring card that is pertinent to musculoskeletal manifestations provided by the National Institutes of Health to rate the severity of chronic GVHD. In that section, a scoring system is shown that allows clinicians to rate the severity of joint contractures and to tick a box if polymyositis, peripheral neuropathy and/or myasthenia gravis are present and, if so, to indicate the degree of severity. Besides evaluating for joint contractures, clinicians caring for patients with musculoskeletal symptoms of chronic GVHD should always monitor a patient's respiratory rate and oxygen saturation since there are reports of respiratory failure as a result of weakness of the respiratory muscles in chronic GVHD. ${ }^{16}$

Another etiology of myositis that was considered in this patient was medication-induced myositis. The patient has been regularly taking sorafenib, a tyrosine kinase inhibitor that has been shown to increase event-free survival in patients younger than 60 years old with FLT3-ITD positive AML albeit with increased toxicity. ${ }^{17}$ While Sorafenib has been reported to cause myositis in at least one case report, however it was not believed to be the culprit in this patient's case since the patient had been taking the drug for several months before the onset of weakness and myopathic symptoms appear to be a relatively rare side effect. ${ }^{18}$ As a result, the patient was instructed to continue taking sorafenib as maintenance for AML.

This patient presented with normal levels of CPK, which may make this presentation unusual since in one review of 36 cases of polymyositis due chronic GVHD, it was reported that the majority of patients presented with elevated CPK and aldolase levels. ${ }^{19}$ Aldolase was not measured in this patient, so unfortunately we cannot know if it was elevated or not. As for the normal level of CPK, while this enzyme typically parallels disease activity in most patients with myositis, it may be normal or only slightly elevated in different types of myositis. ${ }^{20}$ This patient did have a history of acute GVHD, which may or may not be present in common presentations of this disease. In the same case series that reported data on elevated CPK, Couriel et al. found that about half of the patients did not have any history of an episode of acute GVHD prior to their presentation with symptoms of polymyositis. 19 This also means that a significant percentage of patients are likely to present with myositis without any prior indications of graft-versus-host disease.

In muscle biopsies taken in cases of myositis secondary to chronic GVHD, one should expect the same findings as in idiopathic polymyositis, namely perimysial and endomysial infiltrate of $\mathrm{CD}_{4}+$ and CD8 $+\mathrm{T}$ cells in the myofibrils. ${ }^{19}$ This is confirmed in a report by Takahashi et al., who reported $\mathrm{CD}_{4}+$ and $\mathrm{CD} 8+\mathrm{T}$ cells in the perimysial infiltrate and primarily CD8+ infiltrate in the endomysial layer in a 22 year-old patient with polymyositis secondary to chronic GVHD. ${ }^{21}$ Examples of pathology slides showing expected findings can be seen in Figure 2 and Figure 3.

This case shows that it is important for clinicians to be familiar with the phenomenon of polymyositis as a manifestation of chronic GVHD, especially since the etiology of myopathy in these patients may easily be mistaken for something else since they often suffer from multiple co-morbidities and are often on immunosuppressive therapy as well as other medications. Furthermore, more research is necessary to find more effective prophylactic and therapeutic treatments for chronic GVHD that possess a lighter side effect burden with long-term use. Current directions of investigation, which include impeding B cell signaling and germinal center formation, increasing regulatory $T$ cells populations, targeting pro-fibrotic interleukins and $T$ and $B$ cell recruiting chemokines have shown some promise but require more patients and clinical trials to be further validated.'
Figure 2. Lymphocytic Infiltrate in Muscle Tissue.

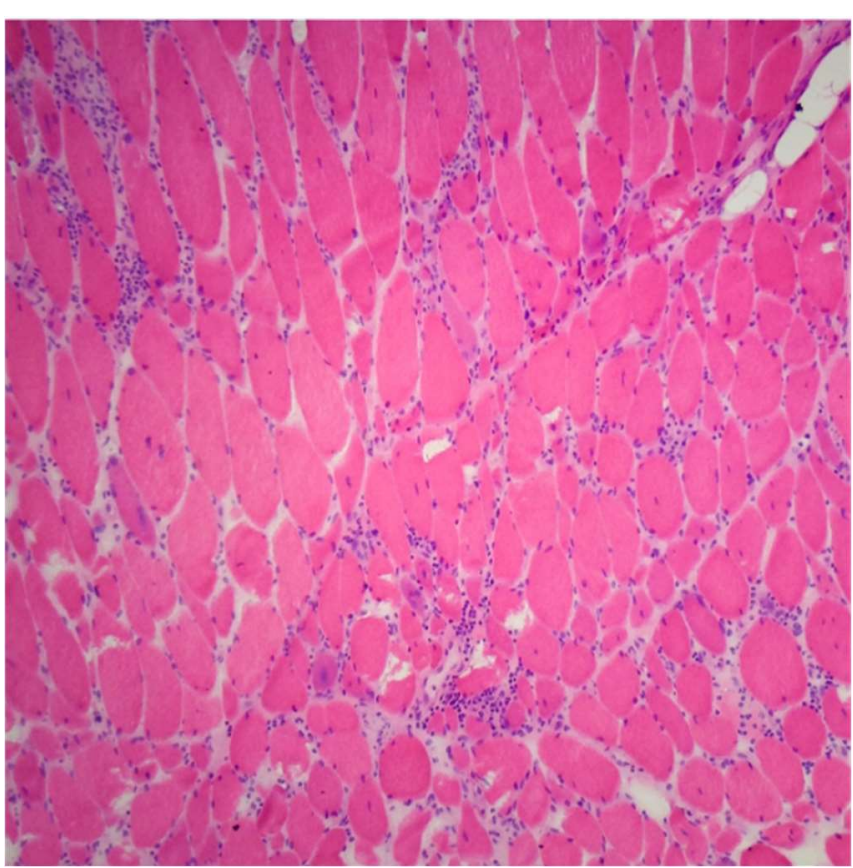

Legend; An HCtE section of a muscle tissue showing infiltration of lymphocytes as well as myopathic features including smaller, rounded myofibers with increased internal nuclei. Courtesy of Meggen Walsh, D.O., M.S.-P.A. ${ }^{22}$

Figure 3. CD8+ T Cell Infiltrate in Muscle Tissue

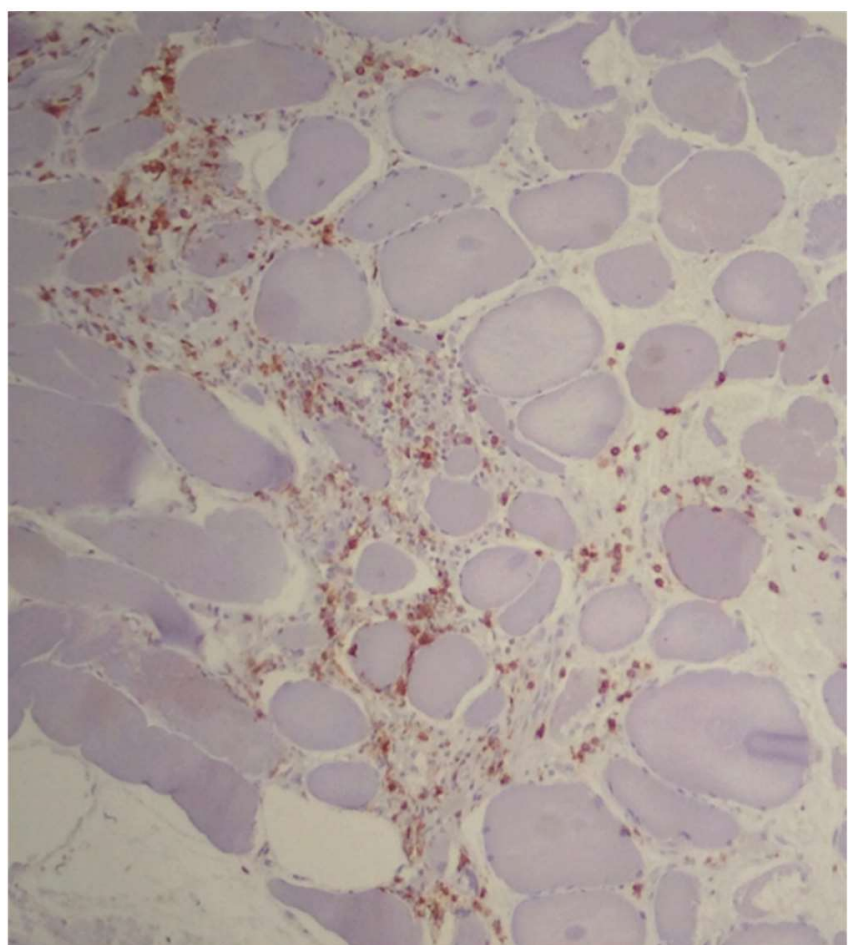

Legend: Immunostaining of muscle from HCAE section showing that infiltrate is composed of $\mathrm{CD}_{3}+\mathrm{CD} 8+\mathrm{T}$ cells. Courtesy of Meggen Walsh, D.0., M.S.-P.A. ${ }^{22}$ 


\section{References}

1. Zeiser R, Blazar BR. Pathophysiology of chronic graft-versus-host disease and therapeutic targets. N Engl J Med. 2017 Dec 28;377(26):2565-79.

2. Lee SJ. Classification systems for chronic graft-versus-host disease. Blood. 2017 Jan 5;129(1):30-7.

3. Zeiser R, Blazar BR. Acute graft-versus-host disease - Biologic process, prevention, and therapy. N Engl J Med. 2017 Nov 30;377(22):2167-79.

4. Filipovich AH, Weisdorf D, Pavletic S, Socie G, Wingard JR, Lee SJ, et al. National Institutes of Health Consensus Development Project on criteria for clinical trials in chronic graft-versus-host disease: I. diagnosis and staging working group report. Biol Blood Marrow Transplant. 2005 Dec;11(12):945-56.

5. Veltri L, Regier M, Cumpston A, Leadmon S, Tse W, Craig M, et al. Incidence and pattern of graft-versus-host disease in patients undergoing allogeneic transplantation after nonmyeloablative conditioning with total lymphoid irradiation and antithymocyte globulin. Bone Marrow Res. 2013;2013:414959.

6. Lee SE, Cho BS, Kim JH, Yoon JH, Shin SH, Yahng SA, et al. Risk and prognostic factors for acute GVHD based on NIH consensus criteria. Bone Marrow Transplant. 2013 Apr;48(4):587-92.

7. Center for International Blood and Marrow Transplant Research. Total Number of HCTs Performed in the United States and Reported to Center for International Blood and Marrow Transplant Research - By Donor Type: 2013-2017. Health Resources and Services Administration. Available from: https://bloodstemcell.hrsa.gov/sites/default/files/bloodstemcell/data/transplan t-activity/transplants-donor-type.pdf. Cited Mar 16, 2020.

8. Shlomchik WD. Graft-versus-host disease. Nat Rev Immunol. 2007 May;7(5):34052.

9. Cooke KR, Luznik L, Sarantopoulos S, Hakim FT, Jagasia M, Fowler DH, et al. The Biology of Chronic Graft-versus-Host Disease: A Task Force Report from the National Institutes of Health Consensus Development Project on Criteria for Clinical Trials in Chronic Graft-Versus-Host Disease. Biol Blood Marrow Transpl. 2017 Feb;23(2):211-34.

10. Vogelsang GB. How I treat chronic graft-versus-host disease. 2001 Mar 1;97(5):1196-201.

11. El-Gamal MI, Anbar HS, Yoo KH, Oh C-H. FMS Kinase Inhibitors: Current Status and Future Prospects. Med Res Rev. 2013 May;33(3):599-636.
12. Stevens AM, Sullivan KM, Nelson JL. Polymyositis as a manifestation of chronic graft-versus-host disease. Rheumatology. 2003 Jan;42(1):34-9.

13. Parker P, Chao NJ, Ben-Ezra J, Slatkin N, Openshaw H, Niland JC, et al. Polymyositis as a a Manifestation of Chronic Graft-Versus-Host Disease. Medecine (Baltimore). 1996 Sep;75(5):279-85.

14. Furst DE, Amato AA, Iorga ŞR, Gajria K, Fernandes AW. Epidemiology of adult idiopathic inflammatory myopathies in a U.S. managed care plan. Muscle and Nerve. 2012 Jan;45(5):676-83.

15. Jagasia MH, Greinix HT, Arora M, Williams KM, Wolff D, Cowen EW, et al. National Institutes of Health Consensus Development Project on Criteria for Clinical Trials in Chronic Graft-versus-Host Disease: I. The 2014 Diagnosis and Staging Working Group Report. Biol Blood Marrow Transplant. 2015 Mar;21(3):389-401.

16. Stephenson AL, Mackenzie IRA, Levy RD, Road J. Myositis associated graft-versushost-disease presenting as respiratory muscle weakness. Thorax. 2001 Jan;56(1):82-4

17. Röllig C, Serve H, Hüttmann A, Noppeney R, Müller-Tidow C, Krug U, et al. Addition of sorafenib versus placebo to standard therapy in patients aged 60 years or younger with newly diagnosed acute myeloid leukaemia (SORAML): A multicentre, phase 2, randomised controlled trial. Lancet Oncol. 2015 Dec;16(16):1691-9.

18. Diaz-Sanchez A, Rodriguez-Salas N, Aramendi T, Balbin E. Myositis due to Sorafenib intake in a patient with hepatocellular carcinoma. Dig Liver Dis. 2011 Jan;43(4):333-4.

19. Couriel DR, Beguelin CZ, Giralt S, De Lima M, Hosing C, Kharfan-Dabaja MA, et al. Chronic graft-versus-host disease manifesting as polymyositis: An uncommon presentation. Bone Marrow Transplant. 2002 0ct;30(8):543-6.

20. Dalakas MC. Inflammatory muscle diseases. N Engl J Med. 2015 Apr;372(18):173447.

21. Takahashi K, Kashihara K, Shinagawa K, Yoshino T, Abe K, Harada M. Myositis as a manifestation of chronic graft-versus-host disease. Intern Med. 2000 Jun;39(6):482-5.

22. Walsh M. Polymyositis [Internet]. Pathology0utlines.com. 2016. Available from: http://www.pathologyoutlines.com/topic/musclepolymyositis.html. Cited Mar 19,2020

\section{Acknowledgments}

I would like to thank the Neurology Department at Beilinson Rabin Medical Center in Petah Tikva, Israel for providing access to patient information for the purposes of creating this manuscript.

\section{Conflict of Interest Statement at Funding}

The Authors have no funding, financial relationships or conflicts of interest to disclose.

\section{Author Contributions}

Conceptualization: DBN. Methodology: DBN. Software: DBN. Validation: DBN. Formal Analysis: DBN. Data Curation: DBN. Investigation: DBN. Resources: DBN. Writing - Original Draft: DBN. Writing - Review at Editing: DBN. Visualization: DBN. Supervision: DBN. Project Administration: DBN.

Cite as:

Ben-Nun D. Polymyositis as a Rare Musculoskeletal Manifestation of Chronic Graft-Versus-Host Disease: A Case Report of a 33-Year-Old Patient. Int J Med Students. 2020 Jan-Apr;8(1):36-40.

This work is licensed under a Creative Commons Attribution 4.0 International License

ISSN 2076-6327

This journal is published by the University Library System, University of Pittsburgh as part of the Digital Publishing Program and is co-sponsored by the University of Pittsburgh Press. 